\title{
Estimating the disease burden of ME/CFS in the United States and its relation to research funding
}

\author{
Mary. E. Dimmock ${ }^{1}$, Arthur A. Mirin ${ }^{2}$ and Leonard A. Jason ${ }^{3 *}$ \\ ${ }^{1}$ Independent Researcher, Connecticut, USA \\ ${ }^{2}$ Applied Mathematician, California, USA \\ ${ }^{3}$ Director, Center for Community Research, DePaul University, USA
}

\begin{abstract}
At the National Institutes of Health (NIH), burden of disease is an important factor in funding decisions along with such factors as scientific opportunity, the quality of the science, and the interest of researchers. Recent studies have quantified the burden for a number of diseases in the United States and the NIH has used that information to analyze how its own funding patterns correspond to disease burden. However, the burden of disease has not been quantified for myalgic encephalomyelitis, also called chronic fatigue syndrome (ME/CFS) and is often underestimated due to a lack of research and the misperceptions about the nature of the disease.

Using the limited information in the literature, this paper develops a preliminary estimate of the disease burden of ME/CFS in the United States, using the World Health Organization's Disability Adjusted Life Years (DALY) measure. The ME/CFS DALY estimate is then compared to the NIH's 2013 analysis of research funding versus DALY across other funded diseases in order to estimate a level of funding for ME/CFS that would be commensurate with disease burden.

Even given the limitations arising from sparse data, this analysis demonstrates that federal research funding for this disease is far less than what would be expected by the burden of the disease. We conclude that the annual research funding for ME/CFS would need to increase twenty-five fold or more to be commensurate with disease burden. This level of funding would best leverage the growing interest of researchers and the significant scientific opportunities that exist to understand the pathology of this disease and to advance diagnostics and treatments.
\end{abstract}

\begin{abstract}
Abbreviations: CDC: Centers for Disease Control and Prevention; DW: Disability Weight; DALY: Disability adjusted life years; ME/CFS: Myalgic Encephalomyelitis/Chronic Fatigue Syndrome; NAM: National Academy of Medicine; NIH: National Institutes of Health; QoL: Quality of Life; YLD: Years Lost due to Disability; YLL: Years Lost due to Death
\end{abstract}

\section{Introduction}

As reported in the 2015 report by the National Academy of Medicine (NAM, previously called the Institute of Medicine), ME/CFS is a "serious, chronic, complex, and multisystem disease that frequently and dramatically limits the activities" of patients and in its severe form, "can consume the lives of those whom it afflicts [1]." The NAM report estimated ME/CFS affects between 836,000 and 2.5 million Americans and can leave patients "more functionally impaired than those with other disabling illnesses, including type 2 diabetes mellitus, congestive heart failure, hypertension, depression, multiple sclerosis, and endstage renal disease." NAM also reported that patients seldom recover and most are unable to work, resulting in an economic impact to our country of 18-24 billion dollars a year in lost productivity and medical costs. Yet, in spite of this, the NAM report cited a "paucity of research" and "remarkably little research funding" and noted that the medical community "generally still doubts the existence or seriousness of this disease."

Recognizing the importance of the burden of disease in health decision-making and planning internationally, the World Health Organization has pioneered the use of the Disability Adjusted Life Year, or DALY, as a single measure of disease burden in a population [2]. The DALY includes the burden of disease due to both death and the years of disability associated with the disease. For instance, as noted in an $\mathrm{NIH}$ report on disease burden, some diseases cause premature death, while other diseases cause decades of impairment and debility [3]. At the same time, diseases can also vary in the level of disease severity and thus the disease burden imposed. Further, the burden of a disease can change over time and across countries as new treatments reduce the number of deaths and the level of debility. One example is the introduction of treatments for AIDS, which likely contributed to the $61 \%$ decrease in its disease burden in the U.S. between 1990 and 2010 as noted by Murray in the study on U.S. burden of disease published in 2013 [4]. Countries with less access to these treatments would be unlikely to experience this decrease in disease burden.

Measures like prevalence and numbers of deaths are also sometimes used to assess disease burden. But as noted in a 2011 study by Gillum, DALY is a more accurate measure of disease burden than

Correspondence to: Leonard A. Jason, Ph.D., Professor of Psychology, Director, Center for Community Research DePaul University, 990 W. Fullerton Ave, Suite 3100, Chicago, Il. 60614, USA, Tel: 773-325-2018, E-mail: ljason@depaul.edu

Key words: chronic fatigue syndrome, disability adjusted life years, disease burden, myalgic encephalomyelitis

Received: November 15, 2016; Accepted: December 06, 2016; Published: December 09, 2016 
these measures because it accounts for both the number of years of premature death and the number of years and the level of debility [5]. Even with that, Gillum noted that DALY is not a perfect predictor of NIH spending, accounting for only $33 \%$ of the variance in NIH spending. The reasons that funding might not track disease burden are complex and, as noted by NIH in its 2015 analysis of disease burden and NIH spending, include factors such as scientific merit, scientific opportunity, portfolio balance, and budgetary considerations in addition to public health needs [3].

Even considering these factors, some diseases receive dramatically less funding than would be expected based on disease burden. In an article discussing NIH's 2015 analysis of how its research funding compared to DALY, journalist Carolyn Johnson noted that in 2010, AIDS received $\$ 3.1$ billion in NIH funding while a deadly lung disease with more than six times the disease burden, received only $\$ 118$ million in funding [6]. That deadly lung disease, chronic obstructive pulmonary disease (COPD), is the third leading cause of death and is primarily caused by smoking. As Claiborne Johnston, dean of the Dell Medical School in Austin, Texas, told Johnson, "we tend to underfund things where we blame the victim," and gave as an example the underfunding of liver disease resulting from drinking. As reported by Johnson, the National Institute of Mental Health similarly found that suicide and eating disorders have been underfunded when they assessed spending versus disease burden.

ME/CFS has suffered from a similar neglect. The 2015 NAM report on ME/CFS noted the lack of research funding, the lack of spending outside of the fields of psychiatry and psychology, and the disbelief, stigma, hostility, and lack of clinical care that patients are subjected to by the medical community. The 2015 NIH Pathways to Prevention report noted that current research had neglected the biological factors underlying the disease and that the medical and research communities had allowed "patients to be stigmatized [7]." Seasoned researchers, successful in other fields, have reported difficulties in getting NIH grants for ME/CFS because of this stigma and disbelief; one such researcher said one of the reviewers on one of his applications told him that ME/CFS is a psychosomatic illness [8]. Reflecting this neglect, NIH spending for ME/CFS averaged just \$5M a year between 1995 and 2014 [9]. According to the NIH funding report, ME/CFS is still near the bottom of all diseases funded; in 2014, ME/CFS received less funding than the $\$ 6 \mathrm{M}$ provided for hay fever and significantly less than diseases with similar or lesser disease burden [10]. Further details are provided in Table 1 [10-13].

The NIH analyzed annual funding versus DALY for 69 conditions in the United States in 2013 (Figure 1) [14]. However, the DALY for $\mathrm{ME} / \mathrm{CFS}$ in the U.S. had not been calculated and was not included in that study. An initial estimate of the DALY for ME/CFS is critically needed to highlight the magnitude of underspending relative to disease burden and to also act as a catalyst for the research studies and the changes to clinical care practices, as further detailed below, that are required to more accurately understand the impact and progression of this devastating disease.

Table 1. 2014 NIH Funding and Prevalence for Selected Diseases.

\begin{tabular}{|l|c|c|c|}
\hline Disease area & Funding (Millions) & U.S. Prevalence & \$ Spending per patient \\
\hline HIV/AIDS & $\$ 2,978$ & $1,200,000$ & $\$ 2,482$ \\
\hline Lupus & $\$ 99$ & 350,000 & $\$ 283$ \\
\hline Multiple sclerosis & $\$ 102$ & 400,000 & $\$ 255$ \\
\hline ME/CFS & $\$ 5$ & $1,060,000$ & $\$ 5$ \\
\hline
\end{tabular}

\section{Definition of DALY}

As defined by the World Health Organization, the DALY is derived using either disease incidence or prevalence. The 2013 U.S. burden of disease study by Murray used the prevalence-based method [4], which is also used in this paper. DALY is calculated as the sum of the years lost due to disability (YLD) and the years lost due to death (YLL):

$$
\mathrm{DALY}=\mathrm{YLD}+\mathrm{YLL},
$$

where $\mathrm{YLD}=\mathrm{P}{ }^{\star} \mathrm{DW}$ and $\mathrm{YLL}=\mathrm{N}^{*} \mathrm{~L}[15]$. Here $\mathrm{P}$ is the prevalence, or number of people with the condition in a given year; DW is the disability weight, a number ranging from 0 to 1 that measures the fraction of lost time due to the severity of the condition; $\mathrm{N}$ is the number of deaths due to the condition in that year; and $\mathrm{L}$ is the average loss in life expectancy per death. The ME/CFS DALY had been previously calculated in a 2003 Australian Burden of Disease study by Begg but did not provide disability weights by severity levels, precluding its direct use in this analysis [16].

\section{Estimating YLD}

The quantity YLD involves the prevalence and the disability weight. The 2015 NAM report cites a U.S. prevalence range of 836,000 to 2.5 million. The lower bound of this range reflects a 1999 study by Jason [17]. However, the upper limit of 2.5 million appears to be based on a Centers for Disease Control and Prevention (CDC) report of "CFSlike" illness, defined by the CDC in its CFS Toolkit as having chronic fatigue but not having the other symptoms required for a diagnosis of CFS according to the Fukuda definition $[18,19]$. Because of the quality of its methodological approaches, Jason's 1999 estimate of prevalence rate of $0.42 \%$ is generally accepted as the best estimate of prevalence rate in adults and is the estimate used in this analysis. Based on a U.S. census estimate of $242,470,820$ adults in 2013, this equates to $1,018,377$ adults with ME/CFS [20]. There is no widely accepted estimate of prevalence in children but it is generally considered to be substantially lower than in adults. A 2006 study by Jordan estimates the prevalence in $13-17$ year olds to be $0.181 \%$ [21]. Using the 2013 U.S. census figure for 15-17 year olds and $40 \%$ of the 2013 U.S. census figure for $10-14$ year olds, we estimate that there were 21.35 million children between the ages of 13 and 17, which equates to an estimated 38,643 13 to 17 year olds with ME/CFS [22]. The prevalence of ME/CFS in children younger than 13 is not well established but is assumed to be even lower and is not included in this analysis. Combining adults and 13-17 year olds gives an overall U.S. ME/CFS prevalence of 1.06 million.

Estimates of disability weight (DW) of ME/CFS in the U.S. do not exist. But the disability weight can be estimated from information provided in the 2003 Australian disease burden study [16] and a 2015 Danish study by Hvidberg on quality of life, in which $105 \mathrm{ME} / \mathrm{CFS}$ patients were compared to those with other diseases, using the North Denmark Health Profile 2010 of 23,392 patients as a reference [23]. Both studies use EQ-5D, a standardized instrument used as a measure of health outcome to assess quality of life or disability weight [24]. The Australian study provides disability weights for different levels of severity while the Danish study does not.

The DW used in the 2003 Australian study, first reported for a 1996 Australian disease burden study by Mathers, is listed in the second column of Table 2, broken out by levels of disease severity [25]. However, the Australian study does not provide estimates of the prevalence by severity. Therefore, the estimates of prevalence at different levels of severity are based on rates of disease severity provided 
Funding vs US Burden of Disease - 2013

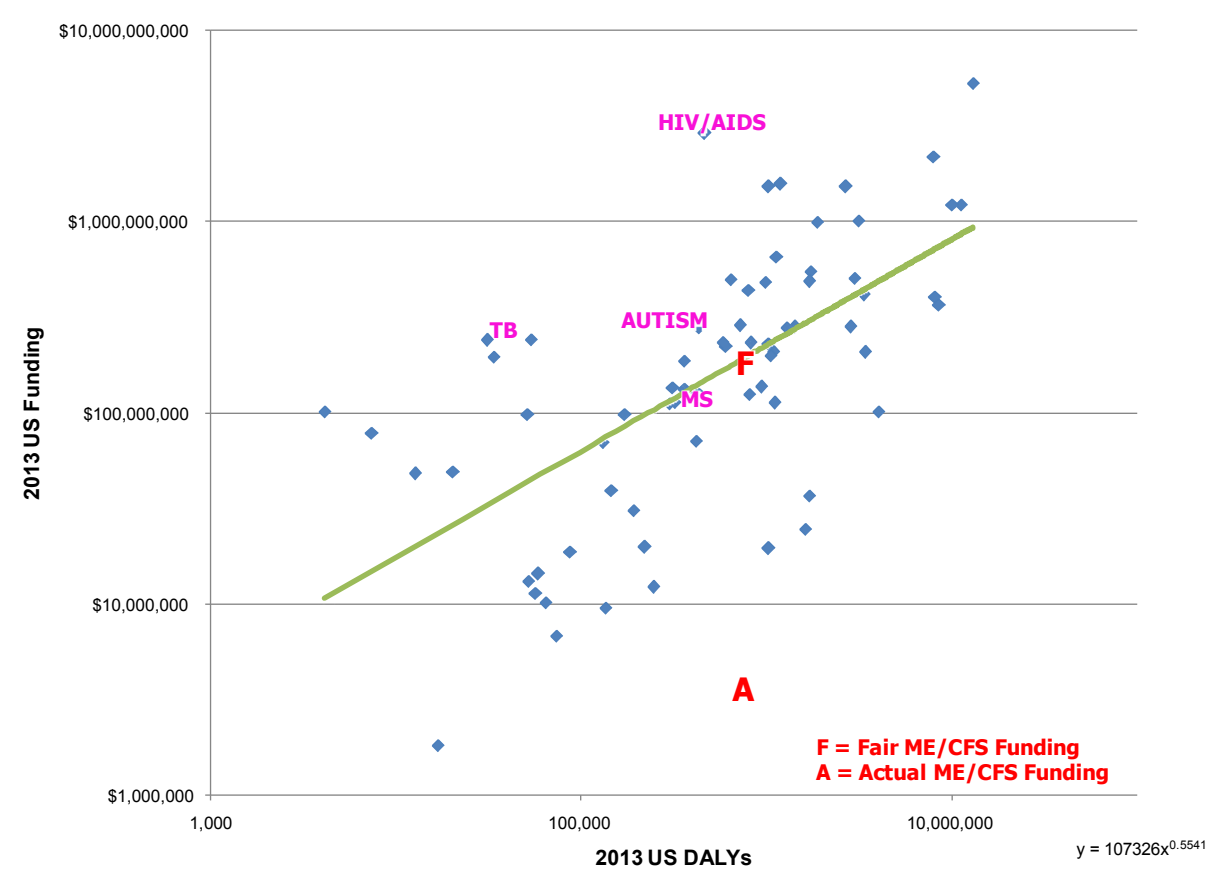

Figure 1. NIH funding versus U.S. burden of disease. Data was downloaded from the website specified in [14].

Table 2. CFS Disability Weight (DW) based on 2003 Australian Study.

\begin{tabular}{|l|c|c|c|}
\hline Severity & DW & Fraction of patients in this group & Contribution to DW \\
\hline Mild & 0.14 & 0.19 & 0.0266 \\
\hline Medium & 0.45 & 0.54 & 0.243 \\
\hline Severe & 0.76 & 0.27 & 0.2052 \\
\hline Totals & & 1 & 0.4748 \\
\hline
\end{tabular}

in other reports. The 2015 National Academy of Medicine report found that $25-29 \%$ of patients are bedbound or homebound [1]. In a 2013 study, Chu reported that as many as $61 \%$ were bedbound on their worst days and that only $13 \%$ could work and some of those only parttime [26]. In 2016, the CDC reported that about $25 \%$ of the patients in its study using multiple ME/CFS specialty clinics were working, an estimate based on a cohort of patients who were well enough to attend one of the few specialty clinics [27]. Based on this information, we estimate that $27 \%$ (midrange of NAM estimate) of patients are severely ill, 19\% (average of Chu's and CDC's estimates) are mildly ill, and the remaining $54 \%$ have an illness of medium severity. These estimates result in a combined DW across all levels of severity of 0.4748 (see Table 2).

The second method of estimating disability weight is based on the 2015 Hvidberg study, which uses EQ-5D to compute quality of life for a number of diseases. We derived the disability weight from the adjusted EQ-5D utility score, QoL, based on the assumption that DW $=1-$ QoL $[28,29]$. This results in an estimated disability weight (DW) of 0.44 for ME/CFS. Among the set of diseases studied by Hvidberg, et al., ME/CFS was found to have the lowest adjusted QoL score and thus the largest estimated DW. The disease with the next largest calculated DW was multiple sclerosis, with an estimated DW of 0.27 based on Hvidberg's catalog of EQ-5D scores for chronic conditions in Denmark. This compares well with the disability weight of 0.28 for multiple sclerosis reported by Saloman in another burden of disease study, the 2013 Global Burden of Disease study [30]. However, other diseases, such as schizophrenia, showed poorer agreement between the two studies. The reason for these differences is not clear.

The fact that both the Australian and Danish studies yield comparable results for the DW for ME/CFS is reassuring. However, there is no obvious reason to choose one method over the other. So the results of both studies have been averaged, resulting in a DW of 0.46 . When combined with a U.S. ME/CFS prevalence of $1.06 \mathrm{M}$, this gives an estimated YLD $\left(=\mathrm{P}^{\star} \mathrm{DW}\right)$ of $0.488 \mathrm{M}$.

\section{Estimating YLL}

Estimating YLL for ME/CFS is much more difficult due to a lack of research on the natural progression of ME/CFS, the lack of tracking of $\mathrm{ME} / \mathrm{CFS}$ patient deaths, and the fact that deaths in ME/CFS patients are most typically attributed to other causes and not related to the disease. However, studies by Jason (2006), March (2014), and McManimen (2016) have documented abnormally high levels of death due to cancer, cardiovascular disease, and suicide [31-33]. Jason analyzed a memorial list of 166 deceased individuals and reported that approximately $20 \%$ died of each of these three causes. In the 2014 study, March conducted a survey of 960 patients seen at four ME/CFS specialty clinics and found that of the 59 deaths that had occurred in that group, 19\% died from heart disease, $19 \%$ from suicide, and 38\% from cancer for a total of $76 \%$ of the reported deaths. The McManimen 2016 study used an online survey to collect information on causes of mortality and found that of 56 deaths reported, $27 \%$ were from suicide, $23 \%$ from cardiovascular disease, and $18 \%$ from cancer.

In addition to studies of mortality, the 2014 Bateman study of 960 adults at four ME/CFS specialty clinics showed an increased prevalence of cancer, while a 2012 study by Chang at the National Cancer Institute showed an increased prevalence of Non-Hodgkin lymphoma [34-35]. A 2016 British study by Roberts did not find any increased mortality for any causes except suicide. However, one of the criteria used to select 
patients in this study required only six months of chronic fatigue for which there was no medical explanation [36]. As McManimen noted, use of these criteria could have included patients who do not have ME/ CFS and could have also included more patients suffering instead from a mental illness, either of which could have resulted in skewed findings [33].

In addition to the increased mortality due to these causes, the 2006 Jason and 2016 McManimem studies also reported that these deaths occurred at a significantly earlier age than normal [31,33]. Both Jason and McManimen reported similar mean ages of death for cardiovascular disease (58.7 in 2006 Jason versus 58.8 in McManimen) and suicide (39.3 in Jason versus 41.3 in McManimen) but significantly different ages for cancer (47.8 in Jason versus 66.3 in McManimen).

In this paper, that fraction of deaths due to these three causes that are above the national average for these specific causes will be considered as complications of the disease and counted as part of the YLL, using the information from the review of 960 patients reported in the 2014 Bateman study [34] and the 2014 March study [32]. The number of years lost due to premature death is estimated using Jason's 2006 study because it is based on a larger sample.

\section{Calculation of the number of deaths}

As noted previously, the primary causes of death among ME/ CFS patients are cancer, heart disease and suicide. For each of these causes, we estimate the death rate in ME/CFS patients (number of deaths divided by size of sample) and compare to the death rate in the population at large. The difference between the two, multiplied by the $\mathrm{ME} / \mathrm{CFS}$ prevalence, is an estimate of the number of Americans with $\mathrm{ME} / \mathrm{CFS}$ who die of that cause as a result of complications of ME/CFS and is included in the YLL.

In Bateman's 2014 study, $15.6 \%$ developed cancer; $7.5 \%$ of those cases were skin cancer, and the remaining $8.1 \%$ were cancers of other types (non-skin). This study reported a high rate of skin cancer versus other cancers compared to the general population, particularly in the clinics in Nevada and Florida. Because of this, we compute separately the contributions of skin and non-skin cancer. Based on national data for 2013, the national prevalence rate for skin cancer was $1.37 \%$ and the prevalence rate for non-skin cancer was $3.087 \%$ while the fraction of patients with cancer who die of cancer was 0.00293 for skin cancer and 0.0581 for non-skin cancer [37-39]. ${ }^{1}$ Based on this data, Bateman's ME/ CFS sample showed an increase in prevalence rate above the national average of $6.13 \%$ for skin cancer $(7.5 \%-1.37 \%)$ and $5.013 \%$ for nonskin cancer (8.1\%-3.087\%). This results in an increased death rate in ME/CFS patients of 0.000180 for skin cancer $\left(0.00293^{\star} 6.13 \%\right)$ and 0.002913 for non-skin cancer $\left(0.0581^{\star} 5.013 \%\right)$, for an increased death

${ }^{1}$ Utilizing the aforementioned references, the National Cancer Institute estimates 14.1M Americans living with cancer in 2013, with $1.034 \mathrm{M}$ having melanoma. The American Cancer Society states that basal and squamous skin cancers are detected in roughly 3.3M Americans each year. Prevalence estimates are difficult to determine, as basal and squamous skin cancers are not reportable according to the American Cancer Society; in the absence of firm prevalence estimates and given that these cancers are generally very treatable, we estimate that all are expeditiously treated, hence a prevalence of $3.3 \mathrm{M}$. The prevalence of other types of skin cancer is low enough to be ignored. Combined with the $1.034 \mathrm{M}$ prevalence of melanoma, that gives a total skin cancer prevalence of $4.33 \mathrm{M}$, hence a non-skin cancer prevalence of $9.77 \mathrm{M}$. Expressed in terms of fraction of 2013 population $(316.5 \mathrm{M})$, the prevalence rate for skin cancer is 0.0137 and for non-skin cancer is 0.03087 . The American Cancer Society estimates $0.580 \mathrm{M}$ U.S. cancer deaths in 2013, 0.0127M due to skin cancer (most from melanoma), leaving $0.5673 \mathrm{M}$ deaths due to non-skin cancer. Hence the fraction of deaths among skin cancer patients is $0.0127 \mathrm{M} / 4.33 \mathrm{M}=0.00293$, and the fraction of non-skin cancer deaths is $0.5673 \mathrm{M} / 9.77 \mathrm{M}=0.0581$. rate of 0.003093 for all cancers. Multiplying this death rate by the ME/ CFS prevalence of $1.06 \mathrm{M}$, the increase in annual cancer deaths in $\mathrm{ME} /$ CFS above the national average is $0.003279 \mathrm{M}$ patients. According to the American Cancer Society, the death rate among the U.S. population as a whole is 0.001833 [40]. Hence, the total yearly death rate due to cancer among the ME/CFS population, including that portion of deaths above the national average, is $0.004926(0.001833+0.003093)$.

In the 2014 March study, 38\% of the deaths were due to cancer and $19 \%$ were due to each of heart disease and suicide [32]. Hence, for heart disease and suicide, we use a death rate of $0.002463(.004926 / 2)$, one half of that for cancer. This represents the total rate of death due to each of these causes from which the increase above the national average must then be determined.

According to the CDC [41], the current heart disease death rate among the general U.S. population is 0.00189 . Hence the increased rate of heart disease death due to ME/CFS is 0.000573 (0.002463 0.00189 ). Multiplied by the ME/CFS prevalence of $1.06 \mathrm{M}$, this results in an increase of $0.00061 \mathrm{M}$ heart disease deaths due to ME/CFS. Given the prevalence in the literature of reports of cardiac disease in ME/CFS patients, this number appears to be unexpectedly low.

According to the CDC [42], the 2014 U.S. suicide rate was 0.000134 . Given the total suicide rate of 0.002463 in ME/CFS patients, this would result in an increase in the yearly death rate due to suicide of 0.00233 (0.002463 - 0.000134). Multiplied by the ME/CFS prevalence of $1.06 \mathrm{M}$, this results in an increased prevalence of $0.00247 \mathrm{M}$ suicides in $\mathrm{ME} /$ CFS over that expected by the national rate of suicide. While suicide is known to be a significant problem in the ME/CFS community, this is a very high rate, equal to 17-18 times the national average. By comparison, the rate of suicide in cancer and multiple sclerosis patients is reported to be twice the national average [43-44]. For depression, one source suggests that the rate of suicide in patients with depression is roughly 6 times the national average [45]. However, a 2000 study by Mayo suggests that between 2 and $9 \%$ of patients with depression commit suicide [46]. Considered in light of the Mayo study, a rate of 0.002463 in ME/CFS patients could be a reasonable estimate, especially given the level of debility and stigma in ME/CFS, and especially given the increased prevalence of both cancer and depression in ME/CFS patients since each of these causes are associated with an increased risk of suicide.

\section{Calculation of the years lost due to premature death}

The 2014 March study did not report the age at which death occurred. Therefore, the 2006 Jason study has been used to estimate the premature years of death. Statistics for Jason's ME/CFS sample are shown in Table 3. Using 2000 as a typical year of death, the expected longevity for a typical 50 year old in that year was 77.8 years for males and 81.8 years for females [47]. This results in lost years of life due to premature death, as shown in the last row of Table 3. Note that this calculation uses the fraction of males and females dying but uses the same mean age of death for both males and females since gender-

Table 3. Years Lost due to Premature Death.

\begin{tabular}{|l|c|c|c|}
\hline & Heart disease & Cancer & Suicide \\
\hline Mean death age & 58.7 & 47.8 & 39.3 \\
\hline Percent male & 34.5 & 17.9 & 17.2 \\
\hline Percent female & 65.5 & 82.1 & 82.8 \\
\hline Years lost (male) & 19.1 & 30.0 & 38.5 \\
\hline Years lost (female) & 23.1 & 34.0 & 42.5 \\
\hline Years lost (average) & 21.7 & 33.3 & 41.8 \\
\hline
\end{tabular}


specific information is not available.

\section{Calculation of the YLL}

The number of increased (not total) deaths (D) due to ME/CFS and its complications is then multiplied by the number of years lost due to premature death (L) to give the YLL for each cause of death.

$$
\begin{array}{ll}
\text { Heart disease: } & \mathrm{D}=0.00061 \mathrm{M} \mathrm{L}=21.7 \text { YLL contrib }=0.0132 \mathrm{M} \\
\text { Cancer: } & \mathrm{D}=0.00328 \mathrm{M} \quad \mathrm{L}=33.3 \text { YLL contrib }=0.1092 \mathrm{M} \\
\text { Suicide: } & \mathrm{D}=0.00247 \mathrm{M} \mathrm{L}=41.8 \text { YLL contrib }=0.1032 \mathrm{M}
\end{array}
$$

The total YLL across all three diseases is $0.226 \mathrm{M}$.

\section{Estimating DALY}

When the YLL of $0.226 \mathrm{M}$ is combined with the YLD of $0.488 \mathrm{M}$, we get a DALY of $0.714 \mathrm{M}$.

\section{Comparison with other diseases}

Noting that the available morbidity statistics on ME/CFS are quite limited, it could be informative to use the YLL information on other diseases, such as multiple sclerosis (MS) that are primarily debilitating rather than death-causing, to estimate YLL in ME/CFS. To the extent that these diseases have a similar death rate and years of life lost, YLL for ME/CFS can be calculated as follows:

$$
\begin{aligned}
& \mathrm{YLL}=(\text { death rate }) * \text { (years lost }){ }^{*} \mathrm{P} \\
& \mathrm{YLL}(\mathrm{ME} / \mathrm{CFS}) / \mathrm{YLL}(\mathrm{A})=\mathrm{P}(\mathrm{ME} / \mathrm{CFS}) / \mathrm{P}(\mathrm{A}) . \\
& \mathrm{YLL}(\mathrm{ME} / \mathrm{CFS})=\mathrm{P}(\mathrm{ME} / \mathrm{CFS}){ }^{*}[\mathrm{YLL}(\mathrm{A}) / \mathrm{P}(\mathrm{A})]=1.06 \mathrm{M}^{*}[\mathrm{YLL}(\mathrm{A}) / \mathrm{P}(\mathrm{A})],
\end{aligned}
$$

where A stands for the other disease. This could be approximately true if the comparison disease primarily causes severe debility but also has a similar profile of numbers and years lost due to premature death.

The previously mentioned 2013 Murray study on the U.S. burden of disease reports MS as having a YLL of $0.0888 \mathrm{M}$ and prevalence of $0.2306 \mathrm{M}$, giving a projected ME/CFS YLL of $0.408 \mathrm{M}$. This YLL figure is roughly twice as large as the $0.226 \mathrm{M}$ previously estimated directly from the limited ME/CFS morbidity data. Without additional data, it is not possible to know if the differences in YLL reflect an underestimate of the number of deaths and years of life lost to premature death in ME/ $\mathrm{CFS}$ or whether the profile of premature death for MS patients is more severe than in ME/CFS patients.

\section{Estimating the Commensurate Level of NIH Research Funding for ME/CFS}

The DALY can be used to estimate the level of NIH research funding that is commensurate with disease burden. To accomplish this, we use Microsoft Excel to approximate the 2013 NIH research funding versus DALY data [14] with a least squares power law approximation:

\section{Annual_Funding $=\$ 0.107326 \mathrm{M} *[\mathrm{DALY}]^{\wedge} 0.5541$}

Results are displayed in Figure 1. Because the data ranges over several orders of magnitude, a power law provides a superior approximation to a straight line. When graphing the logarithm of funding as a function of the logarithm of DALY, the above power law transforms to a straight line, giving us a least squares linear approximation in log-log space. Using the above formula, our estimated ME/CFS DALY of $0.714 \mathrm{M}$ projects an annual funding level of $\$ 188 \mathrm{M}$. Compared to the current $\mathrm{NIH}$ research funding level of $\$ 7 \mathrm{M}$, this represents a 27 -fold increase if $\mathrm{ME} / \mathrm{CFS}$ is going to be funded commensurately with its impact in the
United States.

The estimate of DALY and thus of research funding commensurate with disease burden is based on the best available information about the prevalence of the disease, the level of disability, the numbers of deaths, and the years lost due to premature deaths. However, the available information is very scarce and sometimes conflicting, which can affect the accuracy of the resulting estimates of DALY and commensurate funding. For example, an increase in prevalence of $20 \%$ would result in a DALY of $0.857 \mathrm{M}$ and a corresponding funding level of $\$ 208 \mathrm{M}$, whereas a decrease in prevalence of $20 \%$ would give a DALY of $0.571 \mathrm{M}$ with corresponding funding level of $\$ 166 \mathrm{M}$. For a $20 \%$ increase in prevalence (hence $\mathrm{YLD}=0.586 \mathrm{M}$ ), a $33 \%$ increase in death rate would give YLL of $0.361 \mathrm{M}$ for a DALY of $0.947 \mathrm{M}$ and corresponding funding level of $\$ 220 \mathrm{M}$, whereas a $33 \%$ decrease in death rate would give YLL of $0.181 \mathrm{M}$ for a DALY of $0.767 \mathrm{M}$ and a corresponding funding level of $\$ 196 \mathrm{M}$.

What is particularly notable is that even with these potential inaccuracies in the DALY, the resultant estimates of commensurate funding are far greater than the $\$ 7$ million in NIH funding currently projected for ME/CFS in 2016.

\section{Limitations}

The calculation of DALY for ME/CFS in this study is significantly limited by the lack of research and the poor quality of epidemiological studies assessing the natural progression of the disease, the prevalence of the disease overall and at different levels of severity, the corresponding levels of disability, and the magnitude and timing of premature death due to the disease and its complications. This impacts each of the key factors that are used in the calculation of the DALY:

- Prevalence: Estimates of prevalence in adults vary widely, there are few good estimates of prevalence in children, and there are no well-characterized descriptions and estimates of the different levels of severity or of prevalence estimates broken down by disease severity [48]. A conservative estimate of prevalence, based on the most widely accepted study, has been used to best reflect what is known about the disease but this estimate could be either high or low. The estimates of prevalence for the different levels of severity could also be similarly high or low. This would affect both the YLL and the YLD estimates.

- Disability weights: The disability weights provided in the Australian study and derived from the Danish study are based on EQ5D [16], while the Saloman 2013 Global Burden of Disease disability weights are based on a different methodology [30]. The Australian study did not state how the disability weights were computed from the EQ-5D data, and we inferred a conversion formula for the Danish study [28-29]. Either approach could impact the validity of results and the comparability with disease burden estimates in the Saloman 2013 Global Burden of Disease study and ultimately impact the NIH funding analysis. However, there is good agreement between both methods and also good agreement between the DW calculated for multiple sclerosis from the quality of life estimates in the Danish study and 2013 Global Burden of Disease DW for multiple sclerosis. On the other hand, there is no explanation for the lack of comparability with other diseases, which raises questions about the method of calculating DW that will need to be addressed in future studies.

- $\quad$ Years of premature death: The years lost due to premature death are based on a single study that utilized an analysis of a memorial list that could have produced a skewed result. The other study, using a smaller sample, reported similar findings of years lost due to premature 
death for suicide and heart disease but fewer years lost for cancer. Further, the calculation of normal life expectancy and years lost was based on a 50 year old in year 2000 , which might have introduced some error.

- Causes and numbers of additional deaths due to complications: The numbers of deaths above that seen in the national population are based on very small studies that have significant limitations. In addition, there is also some conflict in the research literature regarding the causes of death, with some ME/CFS studies not reporting increased death due to all of these causes. As with the field in general, some of these differences are due to the use of overly broad definitions, but some differences also reflect the small size of the current studies, the lack of epidemiological research in general, and the lack of understanding about the disease.

Current clinical practices create additional challenges for the calculation of DALY. For instance, according to the 2015 NAM report, 84-91\% of ME/CFS patients have not been diagnosed; this and medical disbelief in the disease has likely contributed to an underreporting of death due to ME/CFS and its complications. In addition, in contrast to how ME/CFS is classified in the International Classification of Diseases (ICD) in other countries, medical electronic records in the United States currently use the same disease classification code (the ICD-10CM code) for both the term "chronic fatigue syndrome" and for the symptom of "chronic fatigue" [49], which precludes differentiating the $\mathrm{ME} / \mathrm{CFS}$ from unspecified chronic fatigue in electronic records. (The term "ME" is still classified as a neurological disease as it is in the World Health Organization's ICD-10 but that diagnosis is seldom given in the U.S.) This electronic records coding practice will make it difficult to leverage the power of electronic medical records in retrospective analyses to understand the causes and ages of premature mortality.

Finally, some may question the inclusion of deaths due to cancer, cardiac disease, and suicide as part of the YLL estimate. However, the literature on the pathology of the disease provides a reasonable basis to postulate how ME/CFS could result in increased mortality due to cancer, cardiovascular disease, and suicide [33]. Including only the fraction of deaths above those seen in the population at large is intended to focus on the complications due to the disease.

To overcome these limitations, epidemiological studies using properly defined patient cohorts must be conducted. This will require additional funding and commitment from both NIH and CDC. Additionally, the misunderstanding in the medical community about the disease, the lack of diagnosis, and the issues with medical coding practices must be addressed so that the disease and the deaths related to the disease are properly tracked in electronic medical records.

Beyond problems with the scarce and conflicting ME/CFS data, we also encountered various uncertainties in assessing deaths due to other causes and estimating the rate of death from these causes in the population at large. For instance, reliable information on the prevalence of skin and non-skin cancers in the general population is difficult to find, thereby affecting our baseline assumptions.

\section{Conclusions}

The goal of this analysis was to provide an initial estimate of the DALY for ME/CFS and use that to estimate the level of NIH funding that would be commensurate with disease burden. Because of the limitations resulting from the lack of research, the lack of funding, lack of diagnosis and poor disease tracking, and the widespread misperceptions about the disease, the resulting estimates of YLD,
YLL, and DALY should be considered rough estimates based on the best data available to the authors at this time. But even with these limitations, this analysis suggests that it is possible to develop a reasonable first approximation of the DALY and use that to gauge the level of commensurate funding. Even with its limitations, this analysis also provides substantial evidence that the level of research funding for $\mathrm{ME} / \mathrm{CFS}$ is far below that expected by its disease burden.

A second goal of this analysis was to highlight the need to do the research necessary to be able to accurately estimate factors like prevalence, the level of disability and the natural progression of the disease. Establishing accurate estimates of death will also require that the issues with medical coding practices and the tracking of ME/CFS deaths noted above be resolved. To ensure that ME/CFS is treated fairly in future NIH funding prioritizations and in public health planning in general, it is essential that an official first estimate of the DALY for ME/CFS be established and then refined as the necessary clinical practices are put in place and the required epidemiological research are undertaken to better understand this disease.

\section{Authorship}

MED and AAM conceptualized the approach to calculating DALY. LAJ provided guidance on the approach and appropriate sources and reviewed the document.

\section{Acknowledgements}

We wish to thank Dr. Lucinda Bateman for helpful feedback on this paper.

\section{Competing interests}

The authors declare that they have no competing interests.

\section{References}

1. National Academy of Medicine, National Academies of Science (2015) Beyond Myalgic Encephalomyelitis/Chronic Fatigue Syndrome: Redefining an Illness.

2. World Health Organization, undated (2016) About the Global Burden of Disease (GBD) Project.

3. Rockey S, Wolinetz C (2015) Burden of Disease and NIH Funding Priorities. Rock Talk. National Institutes of Health.

4. Murray CJ, Atkinson C, Bhalla K, Birbeck G, Burstein R, et al. (2013) The state of US health, 1990-2010: burden of diseases, injuries, and risk factors. JAMA 310: 591-608. [Crossref]

5. Gillum LA, Gouveia C, Dorsey ER, Pletcher M, Mathers CD, et al. (2011) NIH disease funding levels and burden of disease. PLoS One 6: e16837. [Crossref]

6. Johnson CY (2015) Why the diseases that cause the most harm don't always get the most research money. Washington Post.

7. National Institutes of Health (2015) Office of Disease Prevention. Pathways to Prevention Workshop: Advancing the Research on Myalgic Encephalomyelitis/Chronic Fatigue Syndrome.

8. Kitei M (2014) Candid Conversation with Dr. Ian Lipkin. CFSCentral.

9. Dimmock M, Lazell-Fairman M (2015) Thirty Years of Disdain.

10. National Institutes of Health (2016) Estimates of Funding for Various Research, Condition, and Disease Categories (RCDC).

11. Department of Health and Human Services (2014) HIV/AIDS 101: U.S. Statistics

12. American College of Rheumatology (2016) Prevalence Statistics.

13. National Multiple Sclerosis Society (2016) Multiple Sclerosis Association of America. MS Who Gets Multiple Sclerosis.

14. National Institutes of Health (2016) Report on NIH Funding vs. Global Burden of Disease. 
15. World Health Organization (2016) Metrics: Disability-Adjusted Life Year (DALY).

16. Begg S, Vos T, Barker B, Stevenson C, Stanley L, et al. (2007) The burden of disease and injury in Australia 2003. Australian Institute of Health and Welfare. Cat. No. PHE 82.

17. Jason LA, Richman JA, Rademaker AW, Jordan KM, Plioplys AV, et al. (1999) A community-based study of chronic fatigue syndrome. Arch Intern Med 159: 2129-2137. [Crossref]

18. Jason, LA, Torres-Harding S, Njoku MG (2006) The Face of CFS in the U.S. The CFIDS Chronicle.

19. Centers for Disease Control and Prevention (2015) Chronic Fatigue Syndrome, A Toolkit for Providers.

20. IAC Publishing Labs (2016) How many adults live in the U.S.?

21. Jordan KM, Huang CF, Jason LA, Richman J, Mears CJ, et al. (2006) Prevalence of Pediatric Chronic Fatigue Syndrome in a Community-Based Sample. J Chronic Fatigue Syndrome 13: 75-78.

22. United States Census Bureau (2013) Table 1. Population by Age and Sex.

23. Hvidberg MF, Brinth LS, Olesen AV, Petersen KD, Ehlers L (2015) The HealthRelated Quality of Life for Patients with Myalgic Encephalomyelitis / Chronic Fatigue Syndrome (ME/CFS). PLoS One 10: e0132421. [Crossref]

24. EQ-5D (2016) A Standardized Instrument for Use as a Measure of Health Outcome.

25. Mathers C, Vos T, Stevenson C (1999) The burden of disease and injury in Australia (1996). Australian Institute of Health and Welfare. Cat. No. PHE 17.

26. Chu L (2013) US ME/CFS Patient Survey - April to May 2013. Presented at FDA Drug Development Workshop, April 25-26, 2013.

27. Unger B (2016) CDC Grand Rounds: Chronic Fatigue Syndrome: Advancing Research And Clinical Education. Centers for Disease Control and Prevention. March 7, 2016.

28. Ock M, Ahn J, Yoon SJ, Jo MW (2016) Estimation of Disability Weights in the General Population of South Korea Using a Paired Comparison. PLoS One 11: e0162478. [Crossref]

29. Sassi F (2006) Calculating QALYs, comparing QALY and DALY calculations. Health Policy Plan 21: 402-408. [Crossref]

30. Salomon JA, Haagsma JA, Davis A, de Noordhout CM, Polinder S, et al. (2015) Disability weights for the Global Burden of Disease 2013 study. Lancet Glob Health 3: e712-723. [Crossref]

31. Jason LA, Corrdai K, Gress S, Williams S, Torres-Harding S (2006) Causes of Death Among Patients With Chronic Fatigue Syndrome. Health Care for Women International 27: $615-626$.
32. Vallings R, Summary report (p. 7) on Dr. D. March's 2014 IACFS/ME Conference prepublication presentation, The Natural Course of Chronic Fatigue Syndrome: Evidence from a Multi-site Clinical Epidemiological Study.

33. McManimen SL, Devendorf AR, Brown AA, Moore BC, Moore JH, et al. (2016) Mortality in patients with myalgic encephalomyelitis and chronic fatigue syndrome. Fatigue: Biomedicine, Health \& Behavior.

34. Bateman L, Darakjy S, Klimas N, Peterson D, Levine SM, et al. (2015) Chronic fatigue syndrome and co-morbid and consequent conditions: evidence from a multi-site clinical epidemiology study. Fatigue: Biomedicine, Health and Behavior 3(1): 1-15.

35. Chang CM, Warren JL, Engels EA (2012) Chronic fatigue syndrome and subsequent risk of cancer among elderly US adults. Cancer 118: 5929-5936. [Crossref]

36. Roberts E, Wessely S, Chalder T, Chang C-K, Hotopf M (2016) Mortality of people with chronic fatigue syndrome: a retrospective cohort study in England and Wales from the South London and Maudsley NHS Foundation Trust Biomedical Research Centre (SLaM BRC) Clinical Record Interactive Search (CRIS) Register. The Lancet 387: 1638-1643.

37. National Cancer Institute (2016) SEER Stat Fact Sheets: Cancer of Any Site.

38. National Cancer Institute (2016) SEER Stat Fact Sheets: Melanoma of the Skin

39. American Cancer Society (2016) Skin Cancer Facts.

40. American Cancer Society (2013) Cancer Facts and Figures.

41. Centers for Disease Control and Prevention (2015) Heart Disease Facts.

42. Centers for Disease Control and Prevention (2015) Suicide and Self-Inflicted Injury.

43. Sharma SP (2008) High Suicide Rate Among Cancer Patients Fuels Prevention Discussions. J National Cancer Inst 100: 1750-1752.

44. Anderson P (2015) Suicide Rate Almost Double in Patients With MS. Medscape.

45. American Foundation for Suicide Prevention (2016) Suicide claims more lives than war, murder, and natural disasters combined.

46. Bostwick JM, Pankratz VS (2000) Affective disorders and suicide risk: a reexamination. Am J Psychiatry 157: 1925-1932. [Crossref]

47. HealthGrove (2016) 50 Year Old Life Expectancy.

48. Brurberg KG, Fønhus MS, Larun L, Flottorp S, Malterud K (2014) Case definitions for chronic fatigue syndrome/myalgic encephalomyelitis (CFS/ME): a systematic review. BMJ Open 4: e003973. [Crossref]

49. Centers for Disease Control and Prevention (2016) International Classification of Diseases, Tenth Revision, Clinical Modification (ICD-10-CM).

Copyright: (C2016 Dimmock ME. This is an open-access article distributed under the terms of the Creative Commons Attribution License, which permits unrestricted use, distribution, and reproduction in any medium, provided the original author and source are credited. 